\title{
Structural Studies of TraG, the Conjugative Entry Exclusion Protein from the F- plasmid
}

\author{
N Bragagnolo ${ }^{1}$, G Audette ${ }^{2}$ \\ ${ }^{1}$ York University, Bolton, ON, ${ }^{2}$ Dept of Chemistry, York Univ, Toronto, ON \\ nickb13@my.yorku.ca
}

The F-plasmid of Escherichia coli is representative of conjugative type IV secretion systems for the transmission of mobile DNA elements in bacteria, a significant contributor to the evolution of antibiotic resistance. One of the largest proteins of this system, TraG consists of a membrane-bound N-terminal domain, and a periplasmic Cterminal domain denoted $\mathrm{TraG}^{*}$. Each domain has its own function; the membrane bound $\mathrm{N}$-terminal domain is involved in F-pilus assembly while $\mathrm{TraG}^{*}$ is bifunctional. In the donor cell, it interacts with TraN within the outer membrane to facilitate mating pair stabilisation. However, TraG* is also essential in preventing redundant DNA transfer through its interaction with a cognate TraS in the inner membrane of the recipient cell when the recipient carries the same plasmid. Thermofluor, circular dichroism and HDX-MS experiments showed N-terminal truncation mutants of $\mathrm{TraG}^{*}$ displayed higher stability and less disordered content relative to full-length $\mathrm{TraG}^{*}$. The $45 \mathrm{~N}$-terminal residues of $\mathrm{TraG}^{*}$ were predicted to be highly dynamic, possibly serving as a flexible linker between two independently functioning domains. Further truncation mutants of TraG* were designed to enable protein crystallisation.

Acta Cryst. (2020). A76, a7 